\title{
miR-92a Suppresses Mushroom Body-Dependent Memory Consolidation in Drosophila
}

\author{
Tugba Guven-Ozkan, ${ }^{1}$ Germain U. Busto, ${ }^{1}$ Jae-Yoon Jung, ${ }^{2}$ Ilaria Drago, ${ }^{1}$ and Ronald L. Davis ${ }^{1}$ \\ https://doi.org/10.1523/ENEURO.0224-20.2020 \\ ${ }^{1}$ Department of Neuroscience, The Scripps Research Institute Florida, Jupiter, FL 33458 and ${ }^{2}$ Department of \\ Pediatrics/Systems Medicine, Stanford University, Stanford, CA 94305
}

\begin{abstract}
MicroRNAs (miRNAs) fine tune gene expression to regulate many aspects of nervous system physiology. Here, we show that miR-92a suppresses memory consolidation that occurs in the $\alpha \beta$ and $\gamma$ mushroom body neurons (MBns) of Drosophila, making miR-92a a memory suppressor miRNA. Bioinformatics analyses suggested that mRNAs encoding kinesin heavy chain 73 (KHC73), a protein that belongs to Kinesin-3 family of anterograde motor proteins, may be a functional target of miR-92a. Behavioral studies that employed expression of khc73 with and without its 3' untranslated region (UTR) containing miR-92a target sites, luciferase assays in HEK cells with reporters containing wild-type and mutant target sequences in the khc73 3'UTR, and immunohistochemistry experiments involving KHC73 expression with and without the wild-type khc73 3'UTR, all point to the conclusion that $k h c 73$ is a major target of miR-92a in its functional role as a miRNA memory suppressor gene.
\end{abstract}

Key words: Memory consolidation; memory suppressor gene; microRNA; kinesin heavy chain

\section{Significance Statement}

Much remains to be learned about how microRNAs (miRNAs) regulate gene expression for cognitive processes such as memory formation. The important questions include which of the many different miRNAs are involved, what are their targets, and what specific aspects of memory formation do they regulate? Here, we show that the miRNA, miR-92a, normally functions to suppress the consolidation of memories by repressing the expression of a specific kinesin molecule, kinesin heavy chain 73 (khc73), in the mushroom body neurons $(\mathrm{MBn})$ of the Drosophila brain.

\section{Introduction}

MicroRNAs (miRNAs) are small non-coding RNAs that regulate gene repression post-transcriptionally. Since their discovery less than two decades ago, miRNAs have been implicated in diverse biological processes and diseases. The miR-17-92 cluster in humans was one of the earliest miRNAs discovered. It is overexpressed in certain types of cancer and, thus, named oncomiR-1 (He et al., 2005). In addition to its function as an oncogene, this cluster of miRNAs has been implicated in development, immune disease, neurodegenerative diseases and aging (Mogilyansky and Rigoutsos, 2013). The nervous system functions of $m i R-17-$ 92 cluster include the regulation of axonal outgrowth in

Received May 26, 2020; accepted July 28, 2020; First published July 31, 2020. The authors declare no competing financial interests. embryonic cortical neurons (Zhang et al., 2013), hippocampal neurogenesis, and modulation of anxiety, depressive-like behavior, and pain in response to nerve injury in adult rodent models (Jin et al., 2016; Sakai et al., 2017).

Six miRNAs comprise the miR-17-92 cluster, with the functions of individual miRNAs in the cluster generally remaining unclear. However, one miRNA in the cluster, miR92a, regulates neuronal progenitor cell divisions and differentiation during the development of the mouse embryonic neocortex and division of Drosophila neuroblasts (Bian et al., 2013; Yuva-Aydemir et al., 2015), suggesting conservation of function. A role for miR-92a in homeostatic synaptic

Author contributions: T.G.-O., G.U.B., and R.L.D. designed research; T.G.-O., G.U.B., and I.D. performed research; T.G.-O., J.-Y.J., I.D., and R.L.D. analyzed data; T.G.-O. and R.L.D. wrote the paper. 
scaling by repressing rat hippocampal GluA1 subunits of AMPA receptors has been reported (Letellier et al., 2014). In addition, the levels of miR-92a cycle in adult Drosophila pigment-dispersing factor (PDF) neurons across day and night, thus underlying the rhythmicity in excitability of these neurons (Chen and Rosbash, 2017).

Here, we show that miR-92a suppresses Drosophila olfactory memory consolidation by constraining the expression of kinesin heavy chain 73 (khc73). khc73 is homologous to the mammalian Kif13b/GAKIN/Kinesin-3 family of proteins that have plus end-directed microtubule motor activity. khc73 is expressed specifically in the nervous system by late stages of Drosophila embryogenesis (Li et al., 1997) and has a role in asymmetric neuroblast division (Siegrist and Doe, 2005). Kinesin family members also participate in the transport of cargo in axons (Horiguchi et al., 2006), maturation of presynaptic release sites and synaptic function (Tsurudome et al., 2010).

Since restricting miR-92a expression enhances memory consolidation, its normal function must be to suppress consolidation. Hence, miR-92a is characterized as a memory suppressor gene, which we define as any gene that enhances memory performance when removed functionally from an organism. We show that the memory suppressor function is specific to a form of consolidated memory named anesthesia resistant memory (ARM) and that this suppressing function maps to the $\alpha \beta$ and $\gamma$ mushroom body neurons (MBn), neurons known to have dominant roles in olfactory memory formation (Heisenberg, 2003; Guven-Ozkan and Davis, 2014). Opposite to miR-92a, we identify khc73 as a memory promoting gene, enhancing memory consolidation when overexpressed. We present other results showing that khc73 is a target of miR-92a. From a broader perspective, our results indicate that memory consolidation requires anterograde motor proteins, presumably by transporting synaptic cargo from the soma to synapses mediating consolidation.

\section{Materials and Methods}

\section{Fly stocks}

Flies were cultured using standard methods. The flies used for miR-92a inhibition (UAS-miR-92aSP) contained

This work was supported by National Institutes of Health Grants 1R35NS097224 (to R.L.D.) and 1P01NS090994 and David Van Vactor (Harvard).

G. U. Busto's present address: Genetics and Development Department, Institute of Human Genetics, Montpellier 34396, France.

Acknowledgements: We thank David Van Vactor (Harvard) and Tudor Fulga (Oxford) for the miRNA sponge lines and Seth Tomchik (Scripps) for D2R mutant stock. We also thank Pejmun Haghighi (Buck), Fen-Biao Gao (UMass), Chris Doe (U Oregon), the Vienna Drosophila RNAi Center (VDRC), the Bloomington Drosophila Stock Center for other fly lines, David Van Vactor and Dennis Wall for their assistance, and Courtney M. MacMullen for technical support.

Correspondence should be addressed to Ronald L. Davis at rdavis@ scripps.edu.

https://doi.org/10.1523/ENEURO.0224-20.2020

Copyright (C) 2020 Guven-Ozkan et al.

This is an open-access article distributed under the terms of the Creative Commons Attribution 4.0 International license, which permits unrestricted use, distribution and reproduction in any medium provided that the original work is properly attributed. two copies of the sponge transgene, one copy on the second chromosome and another on the third. Similarly, the UAS-scrambled control flies carried a double insertion of the transgene on chromosomes 2 and 3 (Loya et al., 2009; Fulga et al., 2015). miR-92a genomic deletions were as described (Yuva-Aydemir et al., 2015). Other UAS transgenic lines included UAS-miR-92a (Yuva-Aydemir et al., 2015), UAS-khc73, UAS-HA-khc73 (Siegrist and Doe, 2005), UASHA-khc73 ${ }^{+3^{\prime} U T R}$ (Tsurudome et al., 2010), VDRC RNAi lines, and UAS-dcr-2 (Dietzl et al., 2007). GAL4 lines used include 1471, c155, c305a, c739, Gad, GH146, MB-GeneSwitch (247-GeneSwitch), MZ604, nSyb, R11D09, R13F02, R25H11, R28H05, R35B12, Repo, TH, and VT64246.

\section{Behavior}

One- to 5-d-old flies were used for standard two-odor discriminative aversive conditioning paradigm (Beck et al., 2000; Busto et al., 2015; Guven-Ozkan et al., 2016). The odorants used were benzaldehyde (ben) and 3-octanol (oct). Memory was tested using a T-maze. For ARM experiments, anesthesia was induced at different time points after conditioning by transferring flies to glass vials and keeping them on ice for 2 min followed by recovery in regular food vials. For gene switch experiments, flies were fed on food containing $200 \mu \mathrm{M}$ RU486 (MifepristoneSigma) to regulate UAS-driven transgene expression. Memory retention, acquisition, odor, and shock avoidance experiments were performed as described (Busto et al., 2015; Guven-Ozkan et al., 2016).

\section{Bioinformatics and statistical analyses}

Putative mRNA targets for miR-92a were predicted using online tools DIANA-microT-CDS (Paraskevopoulou et al., 2013), TargetScan (Lewis et al., 2005; Ruby et al., 2007), and miRecords (Xiao et al., 2009). The pipeline was designed to select the candidate genes with strong prediction by multiple algorithms. Human miR-92a target genes with homology to Drosophila targets were selected using DIOPT (Hu et al., 2011). GraphPad Prism was used for statistical analyses. Two sample, two-tailed Student's $t$ tests were used to compare two conditions. For multiple group comparisons, one-way ANOVA followed by Dunnett's or Bonferroni's post hoc tests were used.

\section{Molecular cloning and mutagenesis}

To generate khc73 luciferase reporter, $\sim 2.7 \mathrm{~kb}$ of $3^{\prime}$ untranslated region (UTR) was PCR amplified from UASkhc73 ${ }^{+3^{\prime} U T R}$ genomic DNA and cloned into psicheck2 vector downstream of Renilla luciferase using the InFusion enzyme from Clontech. Three miR-92a sites were mutagenized sequentially to generate the triple mutant with an ATAAGCT sequence substituted to GCGGTAC. The triple mutant served as a negative control for miR-92a and $\operatorname{miR}-310$ repression.

\section{Luciferase assay}

HEK293T cells were seeded into 96-well microtiter plates $16 \mathrm{~h}$ before transfection. A total of $400 \mathrm{ng}$ of wildtype or triple mutant psiCheck2-khc73 3'UTR constructs 
were transfected using QIAGEN PolyFect Transfection Reagent and a final concentration of $500 \mathrm{~nm}$ of the miRNA mimic (Dharmacon). Drosophila miR-310 served as positive and Caenorhabditis elegans miR-239b as negative controls. Luciferase substrate of the Dual-Glo Luciferase Assay System (Promega) was used to activate firefly signal followed by Stop\&Glo substrate to inhibit firefly and activate Renilla subsequently. A CLARIOstar plate reader from BMG Labtech was used to measure firefly and Renilla luminescence. Renilla luciferase activity was normalized to firefly. Renilla/firefly ratios for miR-92a and miR-310 transfections were normalized to the miR-239b negative control for both wild-type and triple mutant reporters, independently.

\section{Immunohistochemistry}

We followed the protocol described earlier except that we incubated tissue with primary antibodies for $2 \mathrm{~d}$ (Cervantes-Sandoval et al., 2016; Guven-Ozkan et al., 2016). The primary antibodies used were mouse anti-HA (1:500, 16B12 Thermofisher) and rabbit anti-Scribble (1:500; Cervantes-Sandoval et al., 2016). Secondary antibodies include Alexa Fluor 488 goat anti-mouse (1:500) and Alexa Fluor 633 goat anti-rabbit (1:500). Images were obtained using Leica TCS SP8 confocal microscope. Regions of interest (ROIs) were defined around MB horizontal lobes or antennal lobes. Mean signal intensities of maximum projection images for eighth Z-sections $(3 \mu \mathrm{m}$ each) encompassing the anterior/posterior extent of the MBs were measured using ImageJ. Signal from the antennal lobes was subtracted from MB signal for background normalization. Ratios of $\mathrm{HA}$ and Scribble signals were calculated to minimize brain-to-brain variability. Two sample, two-tailed Student's $t$ tests were used to compare scrambled to miR-92aSP brains.

\section{Results}

\section{miR-92a inhibition enhances aversive olfactory memory}

Employing miRNA sponge transgenes offers a versatile approach to inhibit miRNAs in a time and cell specific manner. We previously screened a large collection of Drosophila miRNA sponge transgenic lines for aversive olfactory memory using the pan-neuronal driver, c155GAL4 (Busto et al., 2015, 2016, 2017; Guven-Ozkan et al., 2016). Our primary screen identified miR-92a as a memory suppressor miRNA since we observed elevated memory performance on reducing the expression of miR-92a with two independent UAS-miR-92a sponge transgenes (miR-92aSP). However, further tests failed to consistently reproduce these initial effects (Busto et al., 2015). We reasoned that this variability might be because of weak inhibition, so we tested the effect of expressing two copies of miR-92aSP with the c155-GAL4 driver. The double sponge caused a significant increase in 3-h memory compared with c155>scrambled control flies (Fig. 1A). To confirm that the memory enhancing effects of expressing miR-92aSP are because of reducing miR-92a expression, we tested heterozygous adult miR-92a knock-out flies to
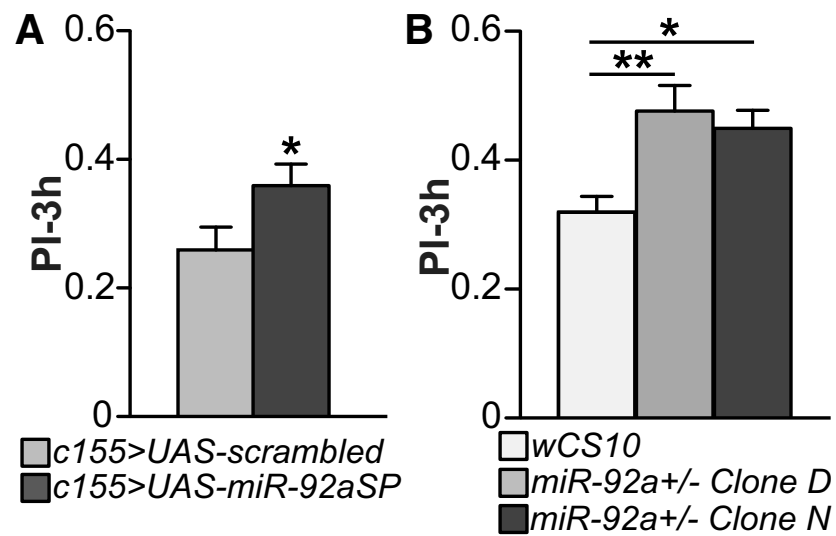

Figure 1. MiR-92a inhibition enhances $3-\mathrm{h}$ olfactory memory. $\boldsymbol{A}$, MiR-92a sponge expression enhances 3-h aversive memory. Pan-neuronal inhibition of miR-92a by expressing a UAS-miR92a sponge (UAS-miR-92aSP) transgene enhances 3-h memory. The UAS-scrambled transgene was used as the control. PI: performance index, Pls are the mean \pm SEM with $n=20$. Twotailed, two-sample Student's $t$ test, $* p<0.05$. B, MiR-92a heterozygous flies exhibit enhanced memory performance. Two lines of a miR-92a deficiency exhibit enhanced Pls compared with a wCS10 control. Pls are the mean \pm SEM with $n=10$. One-way ANOVA followed by Dunnett's post hoc tests, $* p<0.05, * * p<0.01$.

mimic the hypomorphic effects of miR-92aSP knockdown (Fig. 1B). We backcrossed two miR-92a knock-out lines (named D and N; Yuva-Aydemir et al., 2015) into a $w C S 10$ background and employed wCS10 flies as control. Both D and $\mathrm{N}$ clones of the miR-92a+/- heterozygotes showed significantly enhanced memory, similar to that observed with miR-92a sponge (miR-92aSP) flies. Neither miR-92aSP animals nor miR-92a+/- heterozygotes exhibited changes in odor and shock avoidance compared with their respective controls (Extended Data Fig. 1-1).

\section{miR-92a functions in $\mathrm{MB} \alpha \beta$ and $\gamma$ neurons during development and adulthood}

To delineate the subset of neurons in which miR-92a functions as a memory suppressor, we crossed miR92aSP flies with a battery of GAL4 drivers expressed in different neurons of the olfactory nervous system (GuvenOzkan and Davis, 2014). We also included a glia specific GAL4 driver, Repo-GAL4. Among all the drivers tested, inhibition of miR-92a in MBn using R13F02-GAL4 resulted in a memory performance that was significantly elevated compared with the respective scrambled control (Fig. 2A), without altering shock or odor perception (Extended Data Fig. 2-1A). MBn can be classified into three main subtypes based on the trajectory of their axons. Axons of MB $\alpha \beta$ and $\alpha^{\prime} \beta^{\prime}$ neurons bifurcate to form both vertical and horizontal branches whereas $\gamma$ neurons have only a horizontal branch (Crittenden et al., 1998). We used two independent drivers for each MB subtype to inhibit miR-92a in a neuron subtype-specific manner (Fig. 2B) along with R13F02-GAL4 for pan-MBn expression. Inhibiting miR- 

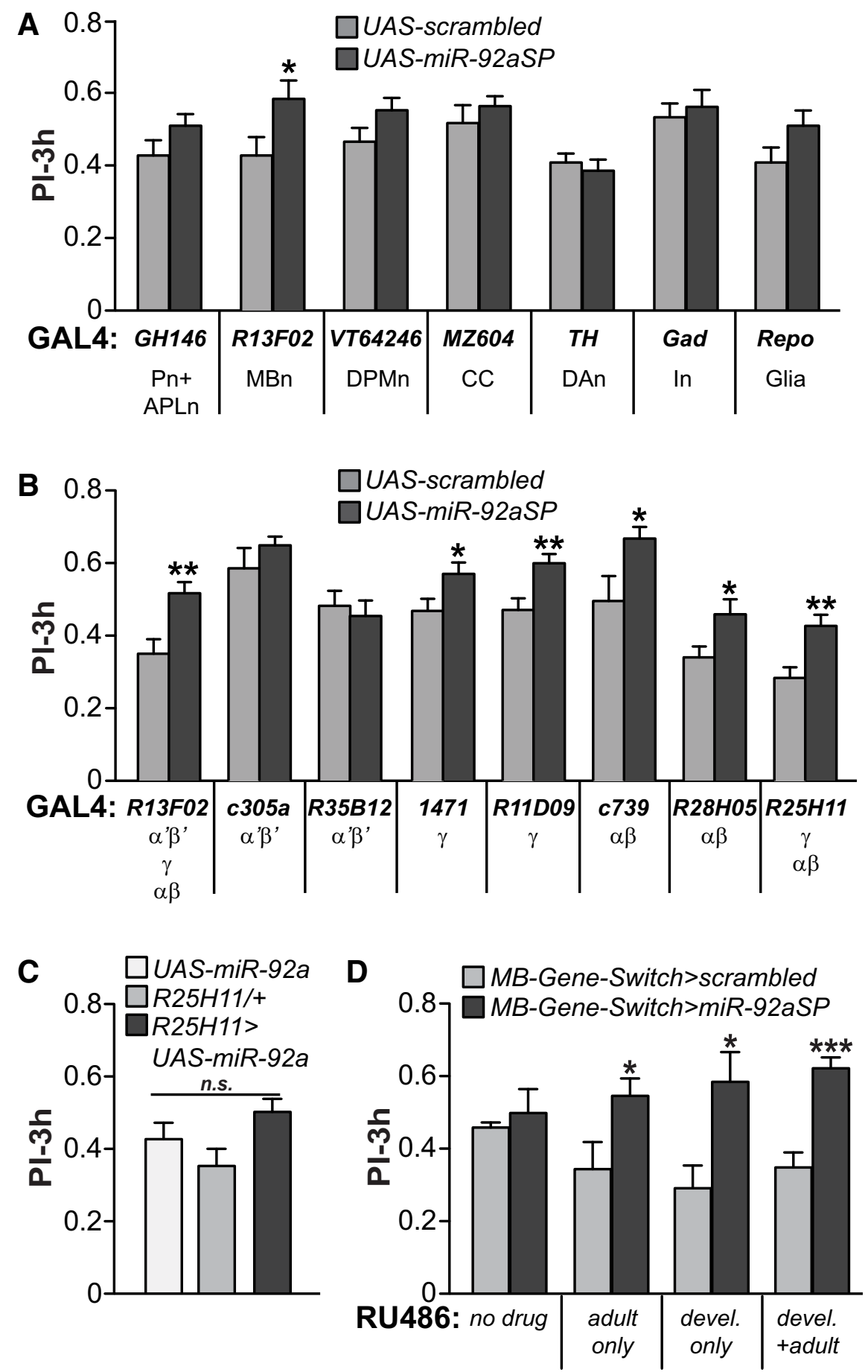

Figure 2. Memory enhancement from inhibiting miR-92a in MBns. $\boldsymbol{A}$, MiR-92a inhibition in mushroom bodies enhances 3-h memory. The UAS-scrambled and UAS-miR-92aSP flies were crossed to a battery of GAL4 lines that drive expression in specific populations of neurons. Most of the drivers promote expression in neurons of the olfactory pathway. The GAL4 drivers used and abbreviations of their expression domains are shown below the bar graph. Note that there is some variation in the scores of the control genotypes because of using different GAL4 drivers. Pn: projection neurons, APLn: anterior paired lateral neuron, DPMn: dorsal paired medial neuron, CC: central complex, DAn: dopaminergic neurons, In: inhibitory neurons. PIs are the mean \pm SEM with $n=12$. Two-tailed, two-sample Student's $t$ tests for each driver expressing miR-92aSP compared with expression of the scrambled control, $* p<0.05$. $\boldsymbol{B}$, MiR-92a spatial mapping to subsets of MBns. Two independent drivers were used for each type of MBn: $\alpha^{\prime} \beta$ ', $\gamma$, and $\alpha \beta$. The GAL4 drivers employed and their expression in the three broad types of MBns are shown below the graph. Pls are the mean \pm SEM with $n=12$. Two-tailed, two-sample Student's $t$ tests for each driver expressing miR-92aSP compared with expression of the scrambled control, $* p<0.05, * * p<0.01$. C , Overexpression of miR-92a does not affect 3-h memory. MiR-92a was overexpressed in $\alpha \beta$ and $\gamma \mathrm{MBns}$ using R25H11-GAL4. Three-hour olfactory memory performance of R25H11-GAL4>UAS-miR-92a flies was 
continued

compared with GAL4-only and UAS-only controls. PIs are the mean \pm SEM with $n=14$. One-way ANOVA followed by Bonferroni's post hoc tests. n.s., not significant. $\boldsymbol{D}$, Memory enhancement occurs from miR-92a inhibition during both development and adulthood as assayed using the Gene-Switch system. Administration of RU486 during development (devel), adulthood (adult), or both (devel + adult) modulates mushroom body expression of miR-92aSP controlled by the MB-Gene-Switch driver. Note that RU treatment either during development or adulthood alters the PIs of the control genotype. PIs are the mean \pm SEM with $n=6-8$. Twotailed, two-sample Student's $t$ tests for each RU486 feeding condition, $* p<0.05, * * * p<0.001$.

92a in MB $\alpha \beta$ and $\gamma$ neurons enhanced memory significantly using 1471-GAL4 and R11D09-GAL4 for $\gamma$ neurons and c739-GAL4 and R28H05-GAL4 for $\alpha \beta$ neurons (Fig. $2 B$ ) without altering odor or shock avoidance (Extended Data Fig. 2-1B,C). R25H11-GAL4, expressed in both $\gamma$ and $\alpha \beta$ neurons, produced the same memory enhancing effect, further strengthening this observation (Fig. 2B; Extended Data Fig. 2-1D). In contrast, miR-92aSP expression in $\alpha^{\prime} \beta^{\prime}$ MBn was without effect (Fig. 2B). To test the effect of increasing miR-92a expression in $\gamma$ and $\alpha \beta$ MBn, we crossed a UAS-miR-92a overexpressing transgene with $R 25 H 11-G A L 4$. This genotype exhibited memory performance at the same level as UAS- and GAL4-only controls (Fig. 2C), indicating that miR-92a is expressed at saturating levels in control flies for memory phenotypes.

We chose to use the Gene-Switch System to dial miR92aSP expression up or down in MBn at different stages of development (McGuire et al., 2004) to delineate developmental versus adult roles. A MBn-specific Gene-Switch GAL4 was crossed to miR-92aSP flies and the progeny were fed the ligand RU486 at different stages to activate the Gene-Switch GAL4. Flies raised on RU486-laced food throughout the lifespan served as a positive control while flies raised on non-supplemented food served as negative control (Fig. 2D). Surprisingly, miR-92a inhibition during development or only during adulthood enhanced 3-h olfactory memory in adult flies compared with flies expressing scrambled sequences. We also tested miR-92aSP memory effect using TARGET system attempting to strengthen our conclusion (McGuire et al., 2004). However, the GAL4 drivers used, in general, failed to reproduce the memory phenotype even when the animals were kept at $30^{\circ} \mathrm{C}$ during both developmental and adult stages (data not shown). The reason for this failure is unknown, although it is possible that the drivers employed were simply not strong enough to overcome the repressive effects of $G A L 80^{t s}$ at the permissive temperature of $30^{\circ} \mathrm{C}$.

\section{miR-92a inhibition enhances intermediate-term memory}

We tested the memory of miR-92aSP flies at different times after aversive conditioning to dissect the phase of memory enhanced by miR-92a inhibition. Memory was not altered at 3-min or 1-h after conditioning using the pan-MBn driver R13F02-GAL4 but was enhanced, relative to control flies, at 2 and $3-h$ after conditioning. This enhancement disappeared by $6-\mathrm{h}$ (Fig. 3A). We replicated and refined this observation by performing the same time course experiment (Extended Data Fig. 3-1A) with drivers that promote expression in the $\gamma(R 11 D 09)$ and the $\alpha \beta$ neurons $(R 28 H 05)$. These experiments reveal clearly that
miR-92a inhibition enhances memory at intermediate time points. To further exclude a possible role for miR-92aSP in memory immediately after conditioning, hidden by a ceiling effect, we tested 3-min memory performance after training flies with varying numbers of shock pulses to titrate training intensity (Fig. 3B; Extended Data Fig. 3-1B). Although performance increased with an increasing number of shock pulses, there was no difference in performance scores between the control and experimental genotype using all three GAL4 drivers (Fig. 3B; Extended Data Fig. 3-1B). These data lead to the strong conclusion that miR-92a inhibition enhances intermediate-term memory without effect on memory acquisition.

\section{miR-92a inhibition enhances memory consolidation}

To further dissect the intermediate-term memory phenotype, we tested whether miR-92a might have a role in
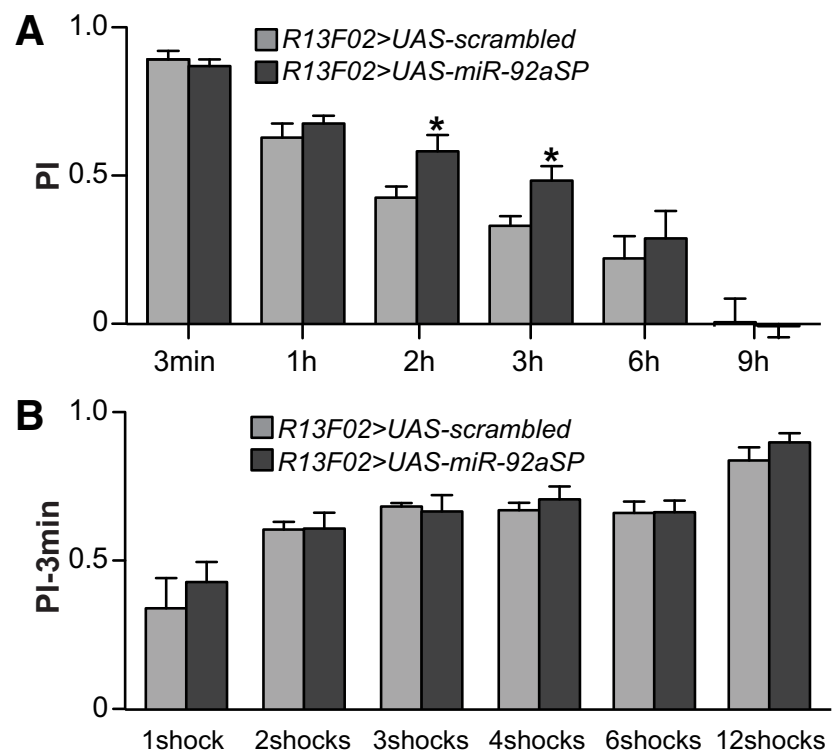

Figure 3. Inhibiting miR-92a enhances intermediate-term memory. $\boldsymbol{A}$, Memory decay in flies expressing miR-92aSP in the mushroom bodies. Three-minute, 1-, 2-, 3-, 6-, and 9-h memory was tested in flies expressing UAS-miR-92aSP or UAS-scrambled under the control of R13F02-GAL4. Pls are the mean \pm SEM with $n=6-8$. Twotailed, two-sample Student's $t$ tests between the groups at each time point, $* p<0.05$. $\boldsymbol{B}$, No effect of miR-92a inhibition on memory acquisition. Three-minute memory of R13F02-GAL4>UAS-scrambled or R13F02-GAL4>UAS-miR-92aSP flies tested after 1, 2, 3, 4,6 and 12 shock-training paradigms. Pls are the mean \pm SEM with $n=6$. Two-tailed, two-sample Student's $t$ tests for each shock group of flies expressing miR-92aSP compared with expression of the scrambled control. No significant differences were found. 

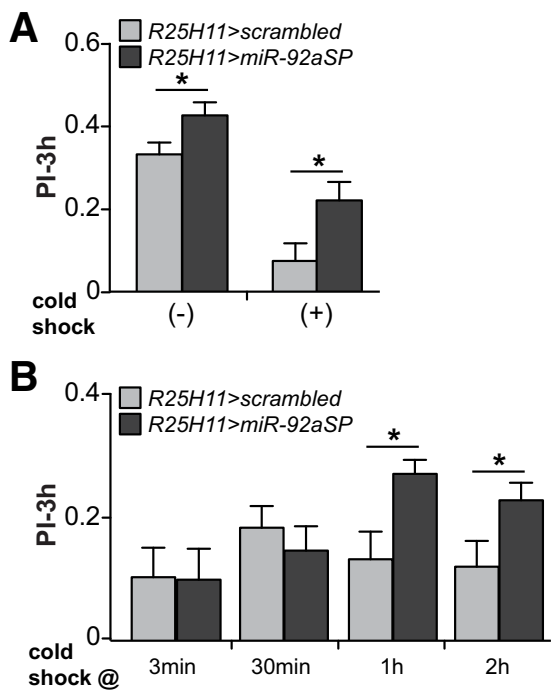

Figure 4. Inhibiting miR-92a enhances memory consolidation. $\boldsymbol{A}$, Three-hour memory performance of R25H11-GAL4>UASscrambled or R25H11-GAL4>UAS-miR-92aSP flies with or without cold shock induced amnesia applied 2-h after aversive training. Cold-shocked flies exhibited increased performance, indicating enhanced ARM. Pls are the mean \pm SEM with $n=14-16$. Two-tailed, two-sample Student's $t$ tests, $* p<0.05$. $\boldsymbol{B}$, Three-hour memory performance of R25H11-GAL4>UASscrambled or R25H11-GAL4>UAS-miR-92aSP flies subjected to cold shock induced amnesia at various time points after aversive training. Pls are the mean \pm SEM with $n=12-16$. Twotailed, two-sample Student's $t$ tests, $* p<0.05$.

memory consolidation. One type of consolidated memory in flies is termed ARM (Quinn and Dudai, 1976; Tempel et al., 1983), traditionally studied by subjecting flies to a 2-min cold shock to trigger amnesia after training and remove anesthesia sensitive memory (ASM). The memory remaining after this insult is then measured at later time points. We measured 3-h memory of R25H11>miR-92aSP flies with or without cold shock given at 2-h after training. Interestingly, miR-92a inhibition enhanced ARM compared with the scrambled control flies indicating a role for miR-92a in consolidation of this form of memory (Fig. 4A). We reproduced and extended this conclusion by assaying ARM in two other genotypes that express miR-92aSP in the $\gamma$ and the $\alpha \beta$ neurons (Extended Data Fig. 3-1C). Moreover, we studied the time course of ARM enhancement by applying the cold shock at various times after training with testing occurring at 3-h. No ARM enhancement was observed with cold shock at 3 or 30-min after training, but cold shock at 1 or 2-h provided the maximal enhancement of ARM (Fig. $4 B$ ). This indicates that miR-92a disruption impacts memory consolidation processes that occur between 30-120-min after conditioning.

\section{miR-92a target prediction pipeline identifies $k h c 73$ as a potential mRNA target of $m i R-92 a$}

To identify miR-92a target mRNAs and their possible roles in memory consolidation, we first used computational prediction software packages including DIANA-microTCDS, Targetscan, and miRecords (Lewis et al., 2005;
Ruby et al., 2007; Xiao et al., 2009; Paraskevopoulou et al., 2013). To extract the strongest predictions and most likely relevant and conserved targets from among the hundreds predicted by each tool, we employed a stringent pipeline that used all three tools (Fig. 5A). DIANAmicroT-CDS ranks predicted genes based on an miTG score with the highest score being 1.0. Thirty-three genes were predicted by this tool with a miTG score of 1.0. TargetScan ranks its predictions based on the number of miRNA sites and also on the degree of seed sequence complementarity to predicted sites. We selected 31 genes with at least two predicted miR-92a binding sites using this tool. Eleven genes were identified in common using these two different algorithms (Fig. 5A). We then determined whether these 11 common genes were predicted by miRecords, which reduced the number of genes of interest to six (Fig. $5 A$ ). We identified human homologs of predicted Drosophila target genes using DIOPT (Hu et al., 2011) and checked whether their mRNA sequences possessed a five or six nucleotide match to the human miR-92a seed sequence. Finally, the five final genes that showed this possible conservation of miRNA:target interaction in humans were tested to see whether they participated in memory formation by RNAi knock-down using a pan-neuronal nSyb-GAL4 driver (Table 1). The top gene identified by this pipeline, identified from impaired memory scores, was kinesin heavy chain gene, khc73 (Fig. 5B). The impaired memory with RNAi knockdown of khc73 is opposite of the memory phenotype observed on expressing miR-92aSP, which is expected, given that reducing the miRNA repressor should increase khc73 expression.

The hypothesis that $k h c 73$ is a target of miR-92a predicts that overexpression of khc73 should enhance memory, mimicking the memory performance of flies with reduced miR-92a levels. Consistent with the prediction, we found that UAS-khc73 overexpression in $\alpha \beta$ and $\gamma \mathrm{MBn}$ using R25H11-GAL4 increased memory performance compared with UAS-only and GAL4-only controls (Fig. $5 C$ ), with shock-avoidance and odor-avoidance controls failing to explain this enhancement (Extended Data Fig. 2-1E). We made two additional observations from behavioral experiments that provide very significant support for this hypothesis. First, the overexpression of khc73 in $\alpha \beta$ and $\gamma \mathrm{MBn}$ enhanced the same form of memory, ARM, as expression of miR-92aSP (Fig. 5D). Since there are very few known genetic insults that enhance ARM, the probability that this represents independent processes is extremely small. Second, we overexpressed UAS-khc73 using the MB-Gene-Switch driver and discovered that increasing khc73 expression during both developmental and adult stages enhances 3-h memory (Fig. 5E). The developmental co-mapping of khc73 overexpression and miR-92a inhibition provides compelling behavioral support for the hypothesis that $k h c 73$ is a primary target of miR-92a largely responsible for its memory suppressor function.

\section{The khc73 3'UTR and miR-92a binding sites are critical for function}

MiRNAs regulate gene expression post-transcriptionally by binding to sequences in the target gene mRNAs. These 

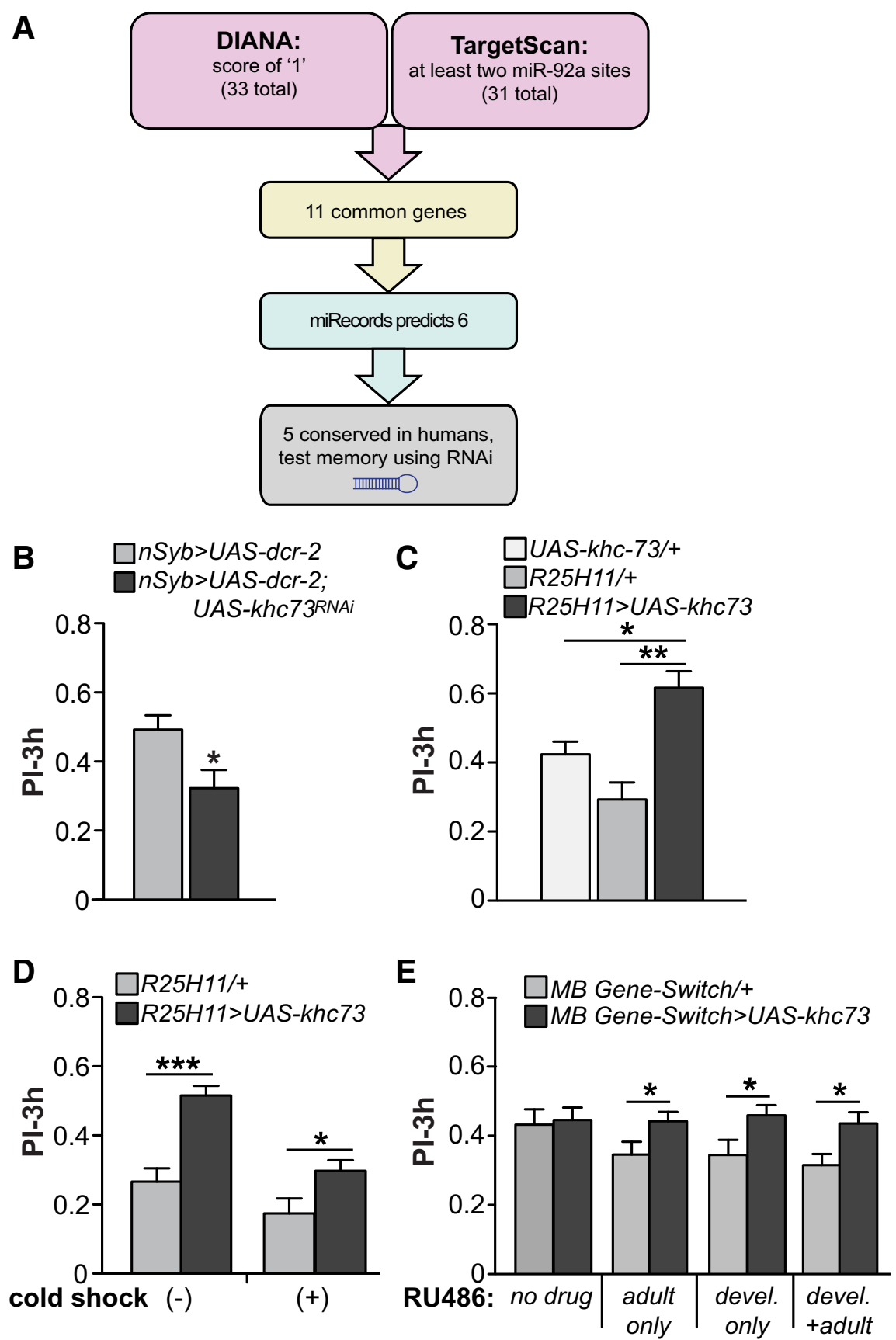

Figure 5. MiR-92a mRNA target prediction. A, Design of the mRNA target prediction pipeline. Top predictions for DIANA and TargetScan have 11 genes in common, six of which are also predicted by miRecords. Five of these potential mRNA targets have human homologs that are predicted targets for human miR-92a. $\boldsymbol{B}$, khc73 RNAi expression produces memory impairment. Only $k h c 73$ of the five candidates (Table 1) significantly impaired 3-h memory using RNAi driven by pan-neuronal $n S y b-G A L 4$. Pls are the mean \pm SEM with $n=10$. Two-tailed, two-sample Student's $t$ tests, $* p<0.05$. C, UAS-khc73 overexpression enhances 3-h memory. Expression of UAS-khc73 driven by R25H11-GAL4 significantly enhanced 3-h memory compared with UAS-only and GAL4-only controls. PIs are the mean \pm SEM with $n=10$. One-way ANOVA followed by Bonferroni's post hoc tests, $* p<0.05, * * p<0.01$. $D$, Overexpressing khc73 using R25H11-GAL4 enhances ARM. Flies were subjected to cold shock 2-h after aversive conditioning and memory was tested at 3-h. Consistent with miR-92a inhibition, overexpressing khc73 enhances ARM. Pls are the mean \pm SEM with $n=12$. Two-tailed, two-sample Student's $t$ tests, $* p<0.05, * * * p<0.001$. $\boldsymbol{E}$, Overexpressing khc73 during development (devel), adulthood (adult), or both (devel + adult) enhances adult memory as assayed using the Gene-Switch system. Administration of RU486 increases mushroom body expression of khc73 controlled by the MB-Gene-Switch driver. Similar to the effect observed in Figure $2 D, \mathrm{RU}$ treatment either during development or adulthood alters the Pls of the control genotype. Pls are the mean $\pm \mathrm{SEM}$ with $n=16-20$. Two-tailed, two-sample Student's $t$ tests for each RU486 feeding condition, $* p<0.05$. 
Table 1: Putative miR-92a target genes

\begin{tabular}{lllll}
\hline Gene name & Flybase ID & RNAi line & PI for $n$ Syb-GAL4 screen & Retest score \\
CG12024 & CG12024 & GD-20143 & $0.45 \pm 0.02$ & $p$ value \\
CG8360 & CG8360 & GD-41643 & $0.40 \pm 0.08$ & \\
cpr50Ca & CG13338 & KK-100317 & $0.64 \pm 0.07$ & $0.56 \pm 0.08$ \\
khc-73 & CG8183 & KK-105984 & $0.38 \pm 0.09$ & 0.15 \\
crebA & CG7450 & KK-110650 & $0.37 \pm 0.06$ & $0.28 \pm 0.07$ \\
& & & & $0.37 \pm 0.08$ \\
\hline
\end{tabular}

Candidate mRNA targets for miR-92a were selected by applying the pipeline described in Figure $5 A$. Five genes were screened for a role in memory formation using an RNAi approach. The five lines from the Vienna Drosophila RNAi left (https://stockcenter.vdrc.at/control/main) that were tested are listed. RNAi lines were crossed to the $n S y b-G A L 4$ driver and tested for 3-h memory with $n=4$. Each individual RNAi line was compared with a daily $n S y b-G A L 4>U A S-d c r-2$ control in the respective GD or KK background. The average performance index for the $n S y b-G A L 4>U A S-d c r-2$ control was $0.36 \pm 0.1$ for the GD control $(n=4)$ and $0.49 \pm 0.04$ for the KK control. Three lines with a trend for a significant effect on memory were retested with $n=6$. Only the khc-73 RNAi line, had a PI significantly lower than the control $(n=10)$. Results shown are the mean \pm SEM. Two-tailed, two-sample Student's $t$ tests, $* p<0.05$.

binding sites are most often located in the 3'UTR of the mRNAs (Miura et al., 2014). Target site identification software predicts three potential miR-92a binding sites in the khc73 3'UTR (Fig. 6A). We first tested the relevance of the khc73 3'UTR to behavior by comparing memory performance of flies that overexpress khc73 from previously characterized transgenes that include the normal $2.9 \mathrm{~kb} \mathrm{khc73}$ 3'UTR and those that include only the 3'UTR from SV40 with its poly A sequences (Siegrist and Doe, 2005; Tsurudome et al., 2010). Strikingly, memory performance was significantly enhanced when khc73 was overexpressed with the SV40 3'UTR, but not when overexpressed with the endogenous 2.9-kb 3'UTR (Fig. 6B). These results support the idea that the khc73 3'UTR provides repressive activity to the memory phenotype and is consistent with the hypothesis that khc73 expression is repressed by miR-92a. To determine whether khc73 is a direct target for miR-92a in vitro, we generated a 3'UTR dual luciferase reporter with khc73 3'UTR using the psiCheck2 vector. The wild-type khc73 3'UTR was cloned downstream of Renilla luciferase so that expression levels may be controlled by regulatory elements present in the khc73 3'UTR (Fig. 6A). The psiCheck2 vector also contains a constitutively expressed firefly luciferase to normalize for transfection efficiency. When the khc73 3'UTRreporter vector was co-transfected with miR-92a RNA mimic, a chemically synthesized miRNA, into HEK293T cells, the Renilla/firefly luminescence ratio was significantly reduced compared with a control mimic (Fig. 6C). Previous studies reported that khc73 3'UTR is repressed at the Drosophila NMJ by the miR-310-313 cluster of miRNAs which belong to the same family of miRNAs as miR-92a (Tsurudome et al., 2010). We thus used one member of the miR-310 cluster as positive control and found that co-transfecting miR-310 mimic resulted in a similar level of repression as miR-92a using the khc73 3'UTR reporter. To further expand these results and map the repression effects to the miR-92a target sequences in the khc73 3'UTR, we generated a mutant luciferase reporter construct, mutating the seed sequences of all three miR-92a/miR-310 sites in the 3'UTR (Fig. 6A). The repressive activity observed with $m i R-92 a$ and $m i R-310$ mimics was not observed using the mutant khc73 3'UTR construct, strongly indicating that $m i R-92 a$ repression occurs through the miR-92a target sites (Fig. 6C). We conclude from our luciferase reporter experiments using cultured cells that miR-92a targets sequences in the wild-type khc73 3'UTR, providing direct control over the expression of $k h c 73$.

We obtained a third line of evidence supporting this conclusion by analyzing $\mathrm{KHC73}$ expression in vivo. We employed HA-tagged, khc73 transgenes with or without the 2.9-kb 3'UTR along with miR-92aSP and quantified expression by immunohistochemistry (Fig. 6D). The UASHA-khc73 transgene carrying only an SV40 3'UTR exhibited robust expression in MBn using R13F02-GAL4 (Fig. $6 D$, left panel). Importantly, the expression was dramatically reduced when the transgene carried the khc73 3'UTR (Fig. 6D, middle panel). This observation is consistent with the behavioral data presented above (Fig. $6 B)$. When we inhibited miR-92a using miR-92aSP transgene, we observed a low but significant level of expression (Fig. 6D), supporting the conclusion that miR$92 a$ represses khc73 through its 3'UTR in vivo. Thus, the behavioral, luciferase reporter experiments and in vivo immunohistochemistry all support the conclusion that the khc-73 3'UTR provides a target for miR-92a repression.

\section{Discussion}

MiRNAs provide impressive regulatory power over gene expression. Individual miRNAs can repress hundreds of mRNAs simultaneously to fine tune the expression of collections of genes post-transcriptionally to regulate biological processes from early development to adult physiology. They function as gene expression rheostats that quickly respond to changing environmental conditions or developmental programs (Ferrante and Conti, 2017). In addition, the expression of miRNAs is altered under circumstances such as stress or disease states. Indeed, miRNAs are widely used as biomarkers for certain diseases and psychiatric conditions and help health care providers in diagnosis of illnesses and in judging efficacy of treatment regimens (Ridolfi and Abdel-Haq, 2017; Lopez et al., 2018). As previously noted, one of the earliest cancer biomarkers identified was the human miR-17-92 cluster of miRNAs (He et al., 2005), for which miR-92a is a member. miR-92a has known roles in nervous system development (Bian et al., 2013; Yuva-Aydemir et al., 2015), adult homeostatic synaptic scaling (Letellier et al., 2014), and neuronal excitability (Chen and Rosbash, 2017). Recently, miR-92a has been identified as a potential plasma biomarker for Alzheimer's patients along with two other miRNAs 
A
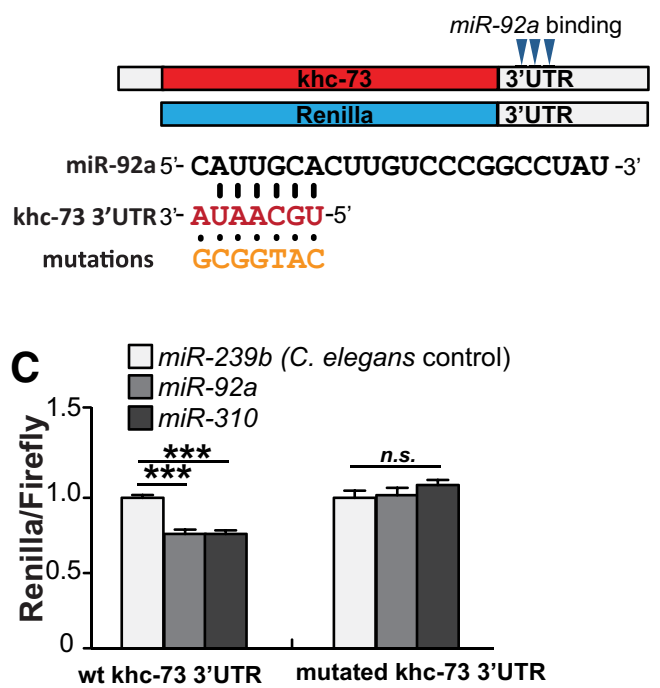

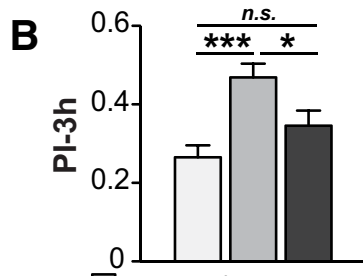

R25H11/+

$\square R 25 H 11>$ UAS-HA-khc73+SV40 3'UTR

R25H11>UAS-HA-khc73+khc73 3'UTR+SV40 $3^{\prime} U T R$

D

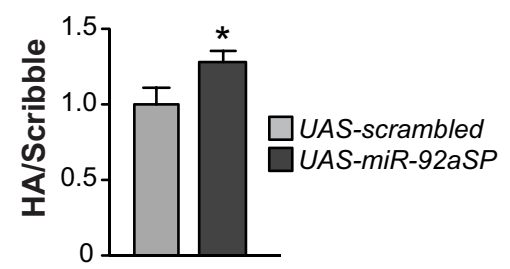

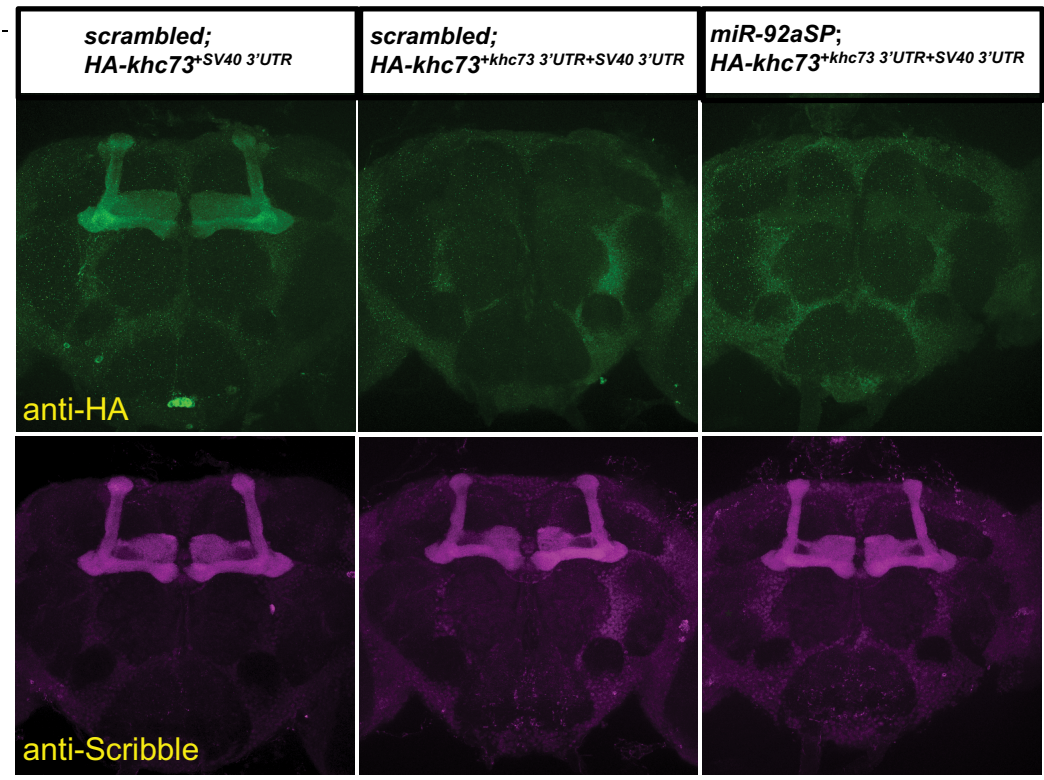

Figure 6. Memory performance of flies overexpressing khc73 with and without the endogenous 3'UTR. A, Three predicted miR-92a binding sites in the endogenous 3'UTR are schematized. Renilla luciferase reporter was cloned upstream of the 2.7-kb khc73 3'UTR and used for the experiment shown in $\boldsymbol{C}$. Nucleotides with predicted miR-92a binding are shown in red font. All three miR-92a sites were altered to generate a mutant khc73 3'UTR that is unresponsive to miR-92a repression (shown with orange font) as a negative control for luciferase experiments. B, Overexpressing khc73 with the endogenous 3'UTR eliminates the memory enhancement gained without the 3'UTR. Expression of HA-tagged khc73 transgenes with or without $2.9 \mathrm{~kb}$ of its own 3'UTR confers normal and enhanced memory performance, respectively. Pls are the mean \pm SEM with $n=14$. One-way ANOVA followed by Bonferroni's post hoc tests, $* p<0.05, * * * p<0.001$. C , Overexpressing miR-92a represses expression of a khc73 3 'UTR luciferase reporter. The Renilla luciferase signal was normalized to firefly luciferase for each transfection. C. elegans miR-239b served as negative and Drosophila miR-310 as positive controls. Renilla/firefly ratios were normalized to negative controls for each reporter construct. Relative repression ratios \pm SEM with $n=9$. One-way ANOVA followed by Bonferroni's post hoc tests, $* * * p<0.001$, n.s., not significant. wt: wild-type. $\boldsymbol{D}, \mathrm{miR}-92 \mathrm{a}$ inhibition derepresses HA-KHC73 signal. HA-Khc73 transgenes with or without the endogenous 2.9-kb 3'UTR element were overexpressed in MBn using the R13F02-GAL4 driver. The presence of the endogenous 3'UTR constraints the HA-KHC73 signal. Very weak signals in the mushroom bodies were detected (middle column) and quantified (bar plot). This weak signal was significantly derepressed when the miR-92aSP was co-expressed (right column). The bar plot shows the quantification of $R 13 F 02>$ scrambled, HA-khc73 + 3'UTR versus R13F02>miR-92aSP, HA-khc73 + 3'UTR brains. ROI was taken from the horizontal lobes by reference to anti-Scribble signal. HA/Scribble ratios were used to minimalize brain to brain variability. Ratios are the mean \pm SEM with $n=26-30$. Two-tailed, two-sample Student's $t$ tests, $* p<0.05$. 
(Siedlecki-Wullich et al., 2019), making miR-92a as a notable molecule for further study in memory formation, cognition and disease.

Our data identify a new function of this conserved miRNA in Drosophila memory consolidation, extending our knowledge of nervous system functions for miR-92a several ways. (1) miR-92a is a memory suppressor gene, functioning in this capacity specifically in the $\alpha \beta$ and $\gamma$ MBn. (2) miR-92a suppresses a specific process in memory formation, the consolidation of early and labile memory into ARM. (3) The memory suppression effect of miR92a appears to occur from effects during development and during adulthood. One caveat of this provisional conclusion is that the lipophilic nature of the Gene-Switch inducer, RU486, may lead to its storage in fat tissues and subsequent release in adult animals to maintain miR92aSP expression (Mao et al., 2004). (4) The memory suppressor functions of miR-92a are mediated largely by the inhibition of the anterograde motor protein, KHC73. (5) khc73, itself, functions in the same $\alpha \beta$ and $\gamma \mathrm{MBn}$ as miR-92a and apparently during both development and adulthood but with opposite effects: promoting consolidation rather than suppressing it.

\section{miR-92a function is very distinct from another suppressor, $m i R-980$}

MiR-92a is not the first identified memory suppressor miRNA. We previously reported that miR-980 functions as a miRNA memory suppressor gene (Busto et al., 2015; Guven-Ozkan et al., 2016). Our findings reveal a diversity of ways by which memory suppressing miRNAs can function. miR-980 suppresses memory broadly from functions in many different cell types in the olfactory nervous system, rather than the two types of MBn identified for miR-92a. This can be explained by the more general function of miR-980: it works by inhibiting the excitable state of neurons, setting a higher threshold for excitation and memory acquisition, rather than suppressing memory consolidation by inhibiting kinesin mediated transport functions as suggested by our current data for miR-92a. Moreover, the functions of miR980 occur during adulthood, rather than having effects during both development and adulthood as suggested by the Gene-Switch experiments with miR-92a. This latter observation is peculiar and poorly understood. It is possible that miR-92a participates in developmental processes from its developmental expression and physiological processes from adult expression. If so, the mRNA targets for miR-92a during development and adulthood could be similar or completely distinct, although the final developmental and adult effects on olfactory memory map to the same $\alpha \beta$ and $\gamma$ $\mathrm{MBn}$.

\section{KHC73 may be the primary target for consolidation effects}

Given the large number of mRNA targets predicted for any given miRNA and the pleasing concept that miRNAs function to control the expression of groups of genes, it is surprising that our data reveal that the miR-92a behavioral effects can largely be explained by the actions of a single

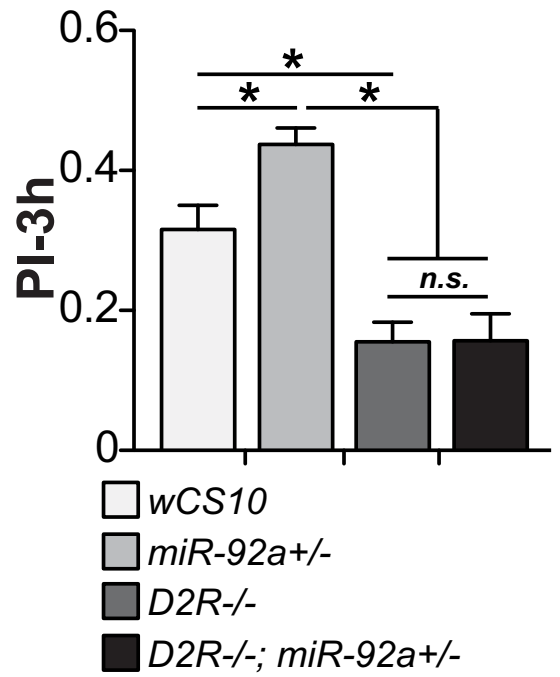

Figure 7. Memory enhancement of miR-92a+/- mutant flies requires $D 2 R$, type 2 dopamine receptor. Three-hour memory scores of miR-92a+/-, D2R-/-, and D2R-/-; miR-92a+/double mutants were tested and compared with wCS10 control using one-way ANOVA followed by Bonferroni's post hoc tests, $* p<0.05$, n.s., not significant. Pls are the mean \pm SEM with $n=20-23$.

target, khc73. However, it may be that different physiological or developmental events regulated by miRNAs are in some cases effected by large groups of genes and others by single or small set of gene targets. In addition, for miRNAs implicated in diverse physiological events, it seems likely that the mRNA targets will be distinct according to the event and the cell types that are involved. Moreover, although the khc73 overexpression memory phenotypes we observed can explain the miR-92a inhibition phenotypes, we cannot exclude the possibility that there may be additional genes regulated by miR-92a that function in memory formation. Furthermore, the khc73 3'UTR harbors predicted sites for other miRNAs. Tsurudome et al. (2010) demonstrated that miR-310 controls khc73 expression at NMJ, distinct from the miR-92a effects in MBn. Thus, this molecular motor protein is regulated by multiple miRNAs in different cell types.

\section{Model for $\boldsymbol{m i R}$-92a and $k h c 73$ function in MBn for suppression of consolidation}

Our data indicate that miR-92a suppresses memory consolidation by restricting the expression of $k h c 73$ in the $\alpha \beta$ and $\gamma \mathrm{MBn}$. Given the role of Kinesin-3 type motor proteins in transport, along with prior studies showing that increased $\mathrm{KHC73}$ expression elevates synaptic transmission (Tsurudome et al., 2010), we propose that upregulated $\mathrm{KHC73}$ levels in miR-92a inhibited flies increases axonal transport and subsequent neurite function and synaptic transmission. Support for this model is found from studies in other systems. Puthanveettil et al. (2008) showed that stimuli leading to consolidated long-term facilitation of the sensory:motor neuron synapse of Aplysia require increased expression of kinesin heavy chain in 
both presynaptic and postsynaptic neurons, and that upregulation of kinesin heavy chain in presynaptic neurons is sufficient for the induction of long-term facilitation. These observations, and ours, support the concept that long-term, or more generally "consolidated plasticity," requires increased kinesin-mediated transport. Our immunohistochemistry experiments reveal that the level of $\mathrm{KHC73}$ when overexpressed with its cognate 3'UTR is surprisingly low compared with when a substitute 3'UTR is used. This suggests that khc73 expression is under tight post-transcriptional control through miRNA regulation. Interestingly, dimerization facilitates fast, processive movement of $\mathrm{KHC73}$ along microtubules (Huckaba et al., 2011). Therefore, the level of KHC73, as regulated by miR-92a, may be the rate-limiting step for fast motor complexes to form. Increased KHC73 levels, because of environmental factors, developmental programs, or other elements that influence miR-92a function would release the brake on cargo transport leading to higher neuronal function and performance.

Our analysis pipeline for identifying miR-92a mRNA targets, in which we used human conservation as a criterion, identified five genes. There are miR-92a seed matches in human Kif13b, which is homologous to Drosophila khc73, two with identical six base pair matches in the coding sequence and one with a 5/6 base pair match in the 3'UTR. It would be interesting to test whether mammalian miR$92 a$ might be involved in memory processes by regulating Kif13b or kinesin genes highly related to Kif13b. Another broad issue for future investigation surrounds the identity of other molecules that may function with miR-92a and khc73 in ARM consolidation. Interestingly, the phenotypes we observed for miR-92a knock-down have an intriguing relationship to those for knock-out of the type 2 dopamine receptor, $D 2 R$. The $D 2 R$ receptor and miR-92a are both involved in ARM consolidation in the same MB subtypes (Scholz-Kornehl and Schwärzel, 2016), with $D 2 R$ knock-out impairing memory while miR-92a inhibition enhances memory. We tested for possible genetic interactions by epistasis experiments. The memory enhancement of miR-92a+/- mutants was abolished and indistinguishable from $D 2 R-/-$ mutants when we tested the double mutants (Fig. 7) suggesting a possible genetic interaction. It would be interesting to explore the mechanistic details of how miR-92a-mediated memory enhancement is dependent on dopamine signaling in the future.

\section{References}

Beck CD, Schroeder B, Davis RL (2000) Learning performance of normal and mutant Drosophila after repeated conditioning trials with discrete stimuli. J Neurosci 20:2944-2953.

Bian S, Hong J, Li Q, Schebelle L, Pollock A, Knauss JL, Garg V, Sun T (2013) MicroRNA cluster miR-17-92 regulates neural stem cell expansion and transition to intermediate progenitors in the developing mouse neocortex. Cell Rep 3:1398-1406.

Busto GU, Guven-Ozkan T, Fulga TA, Van Vactor D, Davis RL (2015) microRNAs that promote or inhibit memory formation in Drosophila melanogaster. Genetics 200:569-580.

Busto GU, Guven-Ozkan T, Chakraborty M, Davis RL (2016) Developmental inhibition of miR-iab8-3p disrupts mushroom body neuron structure and adult learning ability. Dev Biol 419:237-249.
Busto GU, Guven-Ozkan T, Davis RL (2017) MicroRNA function in Drosophila memory formation. Curr Opin Neurobiol 43:15-24.

Cervantes-Sandoval I, Chakraborty M, MacMullen C, Davis RL (2016) Scribble scaffolds a signalosome for active forgetting. Neuron 90:1230-1242.

Chen X, Rosbash M (2017) MicroRNA-92a is a circadian modulator of neuronal excitability in Drosophila. Nat Commun 8:14707.

Crittenden JR, Skoulakis EM, Han KA, Kalderon D, Davis RL (1998) Tripartite mushroom body architecture revealed by antigenic markers. Learn Mem 5:38-51.

Dietzl G, Chen D, Schnorrer F, Su K-C, Barinova Y, Fellner M, Gasser B, Kinsey K, Oppel S, Scheiblauer S, Couto A, Marra V, Keleman K, Dickson BJ (2007) A genome-wide transgenic RNAi library for conditional gene inactivation in Drosophila. Nature 448:151-156.

Ferrante M, Conti GO (2017) Environment and neurodegenerative diseases: an update on miRNA role. Microrna 6:157-165.

Fulga TA, McNeill EM, Binari R, Yelick J, Blanche A, Booker M, Steinkraus BR, Schnall-Levin M, Zhao Y, DeLuca T, Bejarano F, Han Z, Lai EC, Wall DP, Perrimon N, Van Vactor D (2015) A transgenic resource for conditional competitive inhibition of conserved Drosophila microRNAs. Nat Commun 6:7279.

Guven-Ozkan T, Davis RL (2014) Functional neuroanatomy of Drosophila olfactory memory formation. Learn Mem 21:519-526.

Guven-Ozkan T, Busto GU, Schutte SS, Cervantes-Sandoval I, O'Dowd DK, Davis RL (2016) MiR-980 is a memory suppressor microRNA that regulates the autism-susceptibility gene A2bp1. Cell Rep 14:1698-1709.

He L, Thomson JM, Hemann MT, Hernando-Monge E, Mu D, Goodson S, Powers S, Cordon-Cardo C, Lowe SW, Hannon GJ, Hammond SM (2005) A microRNA polycistron as a potential human oncogene. Nature 435:828-833.

Heisenberg M (2003) Mushroom body memoir: from maps to models. Nat Rev Neurosci 4:266-275.

Horiguchi K, Hanada T, Fukui Y, Chishti A (2006) Transport of PIP3 by GAKIN, a kinesin-3 family protein, regulates neuronal cell polarity. J Cell Biol 174:425-436.

Hu Y, Flockhart I, Vinayagam A, Bergwitz C, Berger B, Perrimon N, Mohr SE (2011) An integrative approach to ortholog prediction for disease-focused and other functional studies. BMC Bioinformatics 12:357.

Huckaba TM, Gennerich A, Wilhelm JE, Chishti AH, Vale RD (2011) Kinesin-73 is a processive motor that localizes to Rab5-containing organelles. J Biol Chem 286:7457-7467.

Jin J, Kim SN, Liu X, Zhang H, Zhang C, Seo JS, Kim Y, Sun T (2016) miR-17-92 cluster regulates adult hippocampal neurogenesis, anxiety, and depression. Cell Rep 16:1653-1663.

Letellier M, Elramah S, Mondin M, Soula A, Penn A, Choquet D, Landry M, Thoumine O, Favereaux A (2014) miR-92a regulates expression of synaptic GluA1-containing AMPA receptors during homeostatic scaling. Nat Neurosci 17:1040-1042.

Lewis BP, Burge CB, Bartel DP (2005) Conserved seed pairing, often flanked by adenosines, indicates that thousands of human genes are microRNA targets. Cell 120:15-20.

Li HP, Liu ZM, Nirenberg M (1997) Kinesin-73 in the nervous system of Drosophila embryos. Proc Natl Acad Sci USA 94:1086-1091.

Lopez JP, Kos A, Turecki G (2018) Major depression and its treatment: microRNAs as peripheral biomarkers of diagnosis and treatment response. Curr Opin Psychiatry 31:7-16.

Loya CM, Lu CS, Van Vactor D, Fulga TA (2009) Transgenic microRNA inhibition with spatiotemporal specificity in intact organisms. Nat Methods 6:897-903.

Mao Z, Roman G, Zong L, Davis RL (2004) Pharmacogenetic rescue in time and space of the rutabaga memory impairment by using gene-switch. Proc Natl Acad Sci USA 101:198-203.

McGuire SE, Mao Z, Davis RL (2004) Spatiotemporal gene expression targeting with the TARGET and gene-switch systems in Drosophila. Sci STKE 2004:pl6. 
Miura P, Sanfilippo P, Shenker S, Lai EC (2014) Alternative polyadenylation in the nervous system: to what lengths will 3' UTR extensions take us? Bioessays 36:766-777.

Mogilyansky E, Rigoutsos I (2013) The miR-17/92 cluster: a comprehensive update on its genomics, genetics, functions and increasingly important and numerous roles in health and disease. Cell Death Differ 20:1603-1614.

Paraskevopoulou MD, Georgakilas G, Kostoulas N, Vlachos IS, Vergoulis T, Reczko M, Filippidis C, Dalamagas T, Hatzigeorgiou AG (2013) DIANA-microT web server v5.0: service integration into miRNA functional analysis workflows. Nucleic Acids Res 41: W169-W173.

Puthanveettil SV, Monje FJ, Miniaci MC, Choi YB, Karl KA, Khandros E, Gawinowicz MA, Sheetz MP, Kandel ER (2008) A new component in synaptic plasticity: upregulation of kinesin in the neurons of the gill-withdrawal reflex. Cell 135:960-973.

Quinn WG, Dudai Y (1976) Memory phases in Drosophila. Nature 262:576-577.

Ridolfi B, Abdel-Haq H (2017) Neurodegenerative disorders treatment: the microRNA role. Curr Gene Ther 17:327-363.

Ruby JG, Stark A, Johnston WK, Kellis M, Bartel DP, Lai EC (2007) Evolution, biogenesis, expression, and target predictions of a substantially expanded set of Drosophila microRNAs. Genome Res 17:1850-1864.

Sakai A, Saitow F, Maruyama M, Miyake N, Miyake K, Shimada T, Okada T, Suzuki H (2017) MicroRNA cluster miR-17-92 regulates multiple functionally related voltage-gated potassium channels in chronic neuropathic pain. Nat Commun 8:16079.
Scholz-Kornehl S, Schwärzel M (2016) Circuit analysis of a Drosophila dopamine type 2 receptor that supports anesthesia-resistant memory. J Neurosci 36:7936-7945.

Siedlecki-Wullich D, Català-Solsona J, Fábregas C, Hernández I, Clarimon J, Lleó A, Boada M, Saura CA, Rodríguez-Álvarez J, Miñano-Molina AJ (2019) Altered microRNAs related to synaptic function as potential plasma biomarkers for Alzheimer's disease. Alzheimers Res Ther 11:46.

Siegrist SE, Doe CQ (2005) Microtubule-induced pins/Galphai cortical polarity in Drosophila neuroblasts. Cell 123:1323-1335.

Tempel BL, Bonini N, Dawson DR, Quinn WG (1983) Reward learning in normal and mutant Drosophila. Proc Natl Acad Sci USA 80:1482-1486.

Tsurudome K, Tsang K, Liao EH, Ball R, Penney J, Yang J-S, Elazzouzi F, He T, Chishti A, Lnenicka G, Lai EC, Haghighi AP (2010) The Drosophila miR-310 cluster negatively regulates synaptic strength at the neuromuscular junction. Neuron 68:879893.

Xiao F, Zuo Z, Cai G, Kang S, Gao X, Li T (2009) miRecords: an integrated resource for microRNA-target interactions. Nucleic Acids Res 37:D105-D110.

Yuva-Aydemir Y, Xu XL, Aydemir O, Gascon E, Sayin S, Zhou W, Hong Y, Gao FB (2015) Downregulation of the host gene jigr1 by miR-92 is essential for neuroblast self-renewal in Drosophila. PLoS Genet 11:e1005264.

Zhang Y, Ueno Y, Liu XS, Buller B, Wang X, Chopp M, Zhang ZG (2013) The MicroRNA-17-92 cluster enhances axonal outgrowth in embryonic cortical neurons. J Neurosci 33:6885-6894. 